\title{
INTERACCIONES ENTRE ÍNDICE DE ÁREA FOLIAR, DENSIDAD DEL DOSEL Y PRECIPITACIÓN EFECTIVA DE UN BOSQUE DE POLYLEPIS RETICULATA UBICADO EN UN ECOSISTEMA DE PÁRAMO
}

\author{
INTERACTIONS BETWEEN LEAF AREA INDEX, CANOPY DENSITY AND \\ EFFECTIVE PRECIPITATION OF A POLYLEPIS RETICULATA FOREST LOCATED \\ IN A PARAMO ECOSYSTEM
}

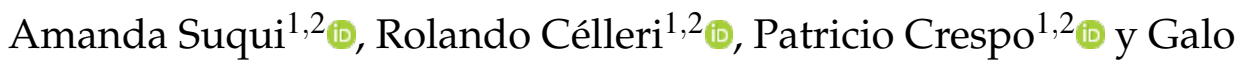 Carrillo-Rojas $* 1,3$ (10)

\footnotetext{
${ }^{1}$ Departamento de Recursos Hídricos y Ciencias Ambientales, Universidad de Cuenca, 010107, Cuenca, Ecuador.

${ }^{2}$ Facultad de Ingeniería, Universidad de Cuenca, 010107, Cuenca, Ecuador.

${ }^{3}$ Facultad de Ciencias Químicas, Universidad de Cuenca, 010107, Cuenca, Ecuador.

*Autor para correspondencia: galo.carrillo@ucuenca.edu.ec
}

Manuscrito recibido el 27 de noviembre de 2020. Aceptado, tras revisión, el 22 de marzo de 2021. Publicado el 1 de septiembre de 2021.

\section{Resumen}

La medición de la cobertura vegetal es fundamental para conocer qué porcentaje de la precipitación queda interceptada sobre la misma. Las técnicas más utilizadas para medir la cobertura in situ son el índice de área foliar (IAF) y la densidad del dosel (DD). Sin embargo, no se ha puesto atención en las diferencias registradas en el uso de las dos técnicas ni cómo estas variables influyen sobre el balance hidrológico particularmente sobre la precipitación efectiva (PE). Por tal motivo, el objetivo del estudio es evaluar la relación entre las mediciones de la cobertura vegetal realizadas por los métodos de IAF y DD e identificar cómo se relacionan con la PE, importante para aplicaciones hidrológicas. El estudio se desarrolló en un bosque de Polylepis reticulata de $15633 \mathrm{~m}^{2}$, ubicado en el Observatorio Ecohidrológico Zhurucay, sur de Ecuador, en un rango altitudinal de 3765 a 3809 m s.n.m. El IAF se midió con el equipo CI-110 Plant Canopy Imager y la DD con un densiómetro esférico, cubriendo un amplio rango de valores de cobertura de dosel. Para medir la PE se instrumentó el sitio de estudio con 9 pluviógrafos. Los resultados indican que el IAF y DD son en promedio $2,43 \mathrm{~m}^{2} \mathrm{~m}^{-2}$ y $88 \%$, respectivamente; cuya relación resulta ser significativa $\left(\mathrm{R}^{2}=0,913 ; \mathrm{p}<0,05\right)$. La PE media anual es de 773,2 mm, que tiende a disminuir con el incremento del IAF y DD; aunque su relación resulta estadísticamente no significativa (valores $\mathrm{p}>0,05$ ). Este estudio muestra la importancia de caracterizar la cobertura vegetal para entender la interacción con la PE.

Palabras clave: Polylepis reticulata, índice de área foliar, densidad del dosel, precipitación efectiva. 


\begin{abstract}
The measurement of vegetation cover is fundamental to quantify the precipitation percentage intercepted by it. The most widely techniques used to measure the cover in situ are the leaf area index (LAI) and the canopy density (CD). However, no attention has been paid to the differences recorded in the use of the two techniques or how these variables influence the hydrological balance on the throughfall (TF). For this reason, the objective of the study is to evaluate the relationship between vegetation cover measurements conducted by the LAI and CD methods and to identify how they relate with the TF, important for hydrological applications. The study was developed in a Polylepis reticulata forest of $15633 \mathrm{~m}^{2}$, located at the Zhurucay Ecohydrological Observatory, south of Ecuador, in an altitudinal range of 3765 to 3809 m.a.s.l. The LAI was measured with the CI-110 Plant Canopy Imager equipment and CD with a spherical densiometer, covering a wide range of canopy cover values. The study site was instrumented with 9 tipping-bucket rain gauges to measure TF. The results indicate that LAI and CD averages are $2.43 \mathrm{~m}^{2} \mathrm{~m}^{-2}$ y $88 \%$ respectively; whose relationship is significant $\left(R^{2}=0.913 ; p<0.05\right)$. Mean annual TF is $773.2 \mathrm{~mm}$, which tends to decrease with the increase of the LAI and CD; although, their relationship is not statistically significant ( $p$-value $>0.05$ ). This study shows the importance of characterizing the vegetation cover to understand the interaction with TF.
\end{abstract}

Keywords: Polylepis reticulata, leaf area index, canopy density, throughfall.

Forma sugerida de citar: Suqui, A., Célleri, R., Crespo, P. y Carrillo-Rojas, G. (2021). Interacciones entre índice de área foliar, densidad del dosel y precipitación efectiva de un bosque de Polylepis reticulata ubicado en un ecosistema de páramo. La Granja: Revista de Ciencias de la Vida. Vol. 34(2):63-79. http:/ / doi.org/10.17163/lgr.n34.2021.04.

IDs Orcid:

Amanda Suqui: http:/ / orcid.org/0000-0002-9776-3033

Rolando Célleri: http:/ / orcid.org/0000-0002-7683-3768

Patricio Crespo: http:/ /orcid.org/0000-0001-5126-0687

Galo Carrillo-Rojas: https:/ / orcid.org/0000-0003-4410-6926 


\section{Introducción}

La cobertura vegetal es un factor clave para comprender los ecosistemas tanto desde el punto de vista hidrológico y ecológico, pues desempeña un papel significativo en las relaciones bosque-agua (Levia y col., 2011), como en la transformación de energía solar en producción primaria (Hernández, Granados y Sánchez, 2003). De hecho, los bosques al presentar una amplia cobertura del dosel y alta rugosidad aerodinámica provocan altas tasas de evaporación potencial (Gerrits, Pfister y Savenije, 2010). Esto quiere decir que la densidad de la cobertura, índice de área foliar, ángulo de inclinación y forma de las hojas inciden sobre los procesos hidrológicos como la intercepción (Crockford y Richardson, 2000; Fleischbein y col., 2005; Gerrits, 2010; Levia y col., 2011), evaporación, precipitación efectiva, e infiltración. La distribución heterogénea del dosel afecta la disposición de agua dentro del área forestal; en otras palabras, en algunos lugares dentro del bosque la lluvia puede alcanzar el suelo en mayor cantidad que en otros, pudiendo exceder la cantidad de precipitación bruta (Gerrits, 2010), debido a que el dosel y la estructura de las ramas canalizan la lluvia formando puntos de goteo que causan mayor intensidad de precipitación efectiva local (Germer, Elsenbeer y Moraes, 2006; Gerrits, 2010).

Uno de los bosques más emblemáticos de los altos Andes son los bosques de Polylepis, los cuales se observan como ecotonos dispersos en el límite arbóreo. Aparecen en un rango altitudinal de 3000 a $5000 \mathrm{~m}$ s.n.m., especialmente en lugares protegidos por rocas o a lo largo de las riveras de los ríos (Domic, Camilo y Capriles, 2014), bajo condiciones ambientales extremas; el clima y la topografía han marcado la existencia de estos bosques como pequeños parches aislados (Renison y col., 2006; Rangel y Arellano, 2010). Bosques que por su alto endemismo son sensibles a cambios (Gareca y col., 2010), encontrándose dentro del grupo de ecosistemas con mayor amenaza (Herzog y col., 2002). Por lo que es importante conocer su aporte en el balance hídrico dentro del páramo, puesto que la vegetación forestal es capaz de interceptar mayor cantidad de agua que el pajonal, inclusive la que proviene de la neblina (Nisbet, 2005).

Es esencial la caracterización del dosel forestal dado que juega un papel fundamental en la parti- ción de la precipitación bruta (precipitación efectiva, escorrentía cortical e intercepción) (Levia y Herwitz, 2005; Johnson y Lehmann, 2006; Park y Cameron, 2008), en el control de la evaporación y almacenamiento de agua (Levia y col., 2011). Además, una descripción detallada del dosel ha facilitado la predicción de las pérdidas de agua por intercepción (Moliĉová y Hubert, 1994). Existen dos métodos básicos de medición: a) el índice de área foliar (IAF) se refiere a la unidad de superficie $\left(\mathrm{m}^{2} \mathrm{~m}^{-2}\right)$ del suelo que se encuentra cubierto por la proyección vertical del dosel o área de las hojas (Jennings, Brown y Sheil, 1999), b) densidad del dosel (DD) o cierre del dosel (\%) es la proporción observada desde un punto único del hemisferio del cielo oscurecido por la vegetación (Jennings, Brown y Sheil, 1999). Las mismas que difieren según el tipo de bosque, densidad, distribución espacial de los árboles, tipo y estructura de copa, estado fenológico de las especies, edad y tipo de manejo (Lieberman, Lieberman y Peralta, 1989; Pukkala y col., 1991). En el caso de bosques de Polylepis estas variables han sido determinadas como parte de investigaciones. Por ejemplo, el IAF ha sido asociada a estudios de caída y descomposición de hojarasca (Pinos y col., 2017). Mientras, la DD ha permitido identificar: el efecto de la cobertura del dosel en la dinámica de las plantas (Cierjacks y col., 2007), su influencia en la avifauna (Tinoco y col., 2013) o la complejidad estructural del paisaje (Renison, Hensen y Suarez, 2011). Puesto que un sin número de estudios se han enfocado en temas como: su distribución en la Cordillera de los Andes (Gosling y col., 2009), historia y causas de fragmentación (Kessler, 2002; Hoch y Körner, 2005; Valencia y col., 2018), características morfológicas (Montalvo y col., 2018), composición florística, y problemas de regeneración (Domic, Camilo y Capriles, 2014; Morales y col., 2018) con la finalidad de entender y conocer las diferencias morfológicas entre especies de Polylepis; así también, conocer las condiciones ecológicas, climáticas en las que estos bosques se desarrollan y actividades que a través del tiempo han causado su fragmentación.

A escala de dosel ocurre dos procesos hidrológicos tan importantes como la precipitación: a) precipitación efectiva (PE) es la cantidad de agua que llega al suelo atravesando el dosel y/o la que cae por goteo después de haber estado en contacto con el follaje (Levia y Frost, 2006), y b) la intercepción es el agua retenida por hojas y ramas de la vegeta- 
ción, reduciendo la cantidad de agua que alcanza el suelo (Gerrits, 2010). Las propiedades del evento de lluvia también inciden sobre estos procesos (Crockford y Richardson, 2000; Murakami, 2006); por ejemplo, una sucesión de eventos con intervalos de periodo seco puede interceptar más agua que una tormenta, ya que parte del agua retenida en el dosel se vacía por evaporación, generando espacio para mayor almacenamiento (Levia y col., 2011). Algunos autores han encontrado que la PE varía de 60 a $95 \%$ de la precipitación bruta (Germer, Elsenbeer y Moraes, 2006; Zimmermann, Wilcke y Elsenbeer, 2007; Berger y col., 2008; Brauman, Freyberg y Daily, 2010), mientras la intercepción puede representar una variación del 10 al 50\% de la misma (Zhang y col., 2006; Roth, Slatton y Cohen, 2007). Esta variabilidad afecta la infiltración, escorrentía, caudal y almacenamiento de agua; procesos consecutivos para completar el ciclo hidrológico (Tsiko y col., 2012). Escasos estudios han mostrado el rol de los bosques de Polylepis en la hidrología como el realizado por Alfaro (2015) en Perú y el de Harden y col. (2013) en Ecuador, que indican la influencia de bosques de Polylepis racemosa (especie introducida y con manejo) sobre la infiltración de agua en el suelo. Investigaciones sobre la precipitación efectiva y la intercepción de agua en el dosel han puesto mayor énfasis en bosques alto andino (Ramos Franco y Armenteras, 2019), bosques montano bajo (Fleischbein y col., 2005; Wullaert y col., 2009), bosques montano tropical (Zimmermann, Wilcke y Elsenbeer, 2007; Gomez y col., 2008) y bosque tropical templado (Oyarzún y col., 2011), facilitando la comprensión del balance hidrológico.

En síntesis, existe muy poca información sobre la relación entre las características del dosel y estas a su vez con la cantidad de agua que alcanza el suelo en bosques altoandinos y más aún en bosques que se encuentran en el límite arbóreo, por lo que se desconoce el rol de la cobertura vegetal de los bosques de Polylepis en los procesos hidrológicos que se dan a escala de dosel. Por este motivo, el presente estudio tiene como objetivo evaluar la relación entre las mediciones de la cobertura vegetal realizadas por los métodos de IAF y DD e identificar como se relaciona con la PE, lo cual es importante para las aplicaciones hidrológicas.

\section{Materiales y Métodos}

\subsection{Sitio de estudio}

El estudio se llevó a cabo en el Observatorio Ecohidrológico Zhurucay donde existe un bosque de Polylepis de $15633 \mathrm{~m}^{2}$, que se encuentra en un rango altitudinal de 3765 a 3809 m s.n.m., con pendientes que varían de 10 a $50 \%$. La especie vegetal dominante es Polylepis reticulata, encontrando otras especies arbóreas como Escallonia myrtiloides, Oreopanax sp., Weinmannia sp., Gynoxys sp., especies de la familia de las Melastomataceae y arbustos como Valeriana sp. Los árboles de Polylepis reticulata pueden alcanzar una altura de $15 \mathrm{~m}$, presentan troncos tortuosos con varias ramificaciones, un diámetro altura al pecho de 33,58 cm y un área basal de 925,64 $\mathrm{cm}^{2}$. Las hojas son alternas que miden hasta $2,5 \mathrm{~cm}$ de largo y crecen conglomeradas en las puntas de las ramas, están conformadas por 3 o 5 foliolos elípticos.

El clima está influenciado por el régimen de humedad del Pacífico y masas de aire continentales que provienen de la cuenca del Amazonas (Córdova, Carrillo y Célleri, 2013). La precipitación interanual se caracteriza por ser altamente uniforme, siendo ligeramente mayor en los meses de Enero a Julio; la precipitación media anual es de $1300 \mathrm{~mm}$ (Ochoa, Crespo y Célleri, 2018). La precipitación ocurre frecuentemente como llovizna que representa el $80 \%$ de los días lluviosos (Padrón y col., 2015). El rango medio de temperatura diaria es de $0,4{ }^{\circ} \mathrm{C}$ a $14,2{ }^{\circ} \mathrm{C}$, con promedio anual de $6,1^{\circ} \mathrm{C}$. La humedad relativa promedio anual es de $93,6 \%$. El nivel de radiación solar es de $4942 \mathrm{MJm}^{-2}$ año $^{-1}$ con una media diaria de $13,73 \mathrm{MJ} \mathrm{m}^{-2}$ día $^{-1}$. La velocidad del viento sigue un patrón estacional con una media mensual de 3,21 m s${ }^{-1}$ para los meses de Octubre a Marzo y 4,77 $\mathrm{m} \mathrm{s}^{-1}$ de Junio a Septiembre (Carrillo y col., 2019). Esta zona presenta una evapotranspiración de referencia anual de $723 \mathrm{~mm}$ a una altitud de 3780 m s.n.m. (Córdova y col., 2015) y una evapotranspiración actual anual (Eta) de $622 \mathrm{~mm}$ (tasa media diaria de 1,7 mm) (Ochoa y col., 2019).

\subsection{Diseño del estudio}

Para la ubicación de los sitios para la medición de la PE, IAF y DD dentro del bosque de Polylepis se realizaron varias actividades: 
1. El área del bosque fue dividida en una grilla de $20 \mathrm{~m} \times 20 \mathrm{~m}$ para determinar en cada punto de intersección el porcentaje de DD (proporción de cielo cubierto por la vegetación) con un densiómetro esférico cóncavo a la altura de los codos (1,20 m sobre el suelo) y a una distancia de $30 \mathrm{~cm}$ del operador, método que se describe en la sección 3.2.

2. Con los valores obtenidos se caracterizó la variabilidad espacial de la DD del bosque. Para identificar la mejor caracterización se usaron varios métodos de interpolación y se analizaron los errores de cada uno; los métodos usados fueron: Kriging ordinario (modelo esférico), ponderación de distancia inversa (IDW) y polígonos de Thiessen.

3. Se identificó la ubicación espacial de 9 sitios de muestreo distribuidos en valores bajos, medios y altos de DD, considerando a su vez el efecto de borde (Figura 1).

\subsection{Medición de la densidad del dosel}

Una vez establecidos los sitios de muestreo para la medición de la PE se midió el porcentaje de DD sobre cada pluviógrafo. Para determinar esta variable se utilizó un densiómetro esférico constituido por un nivel de burbuja y un espejo cóncavo dividido por una grilla de 24 celdas cuadradas que refleja la luz incidente en un ángulo de $180^{\circ}$. Cada lectura consiste en la subdivisión mental de cada celda en 4 cuadrados que se representan mediante un punto imaginario en el centro, dando un total de 96 puntos centrales, que al encontrarse cubiertos por el reflejo de la cobertura vegetal son contabilizados. Se obtuvo un valor promedio de cuatro lecturas por sitio (dirección de los puntos cardinales), el mismo que para obtener el porcentaje de dosel se multiplica por 1,04 (1/96*100) (Lemmon, 1956; Lemmon, 1957; Cook y col., 1995).

\subsection{Medición del índice de área foliar}

La medición de IAF $\left(\mathrm{m}^{2} \mathrm{~m}^{-2}\right)$ se realizó en cada sitio donde fue medida la DD. Se usó el equipo óptico CI-110 Plant Canopy Imager, que consiste de una cámara de 8 megapíxeles equipada con un lente hemisférico (ojo de pez) de un ángulo de $170^{\circ}$. El software se basa en el cálculo de la fracción del cielo visible bajo el dosel utilizando el procedimiento de Gap-Fraction Inversion (Norman y Campbell, 1989) , de acuerdo a tres ecuaciones principales: coeficiente de transmisión para el ingreso de radiación difusa, coeficientes de extinción del dosel (IAF) y el ángulo medio de inclinación del follaje. Las imágenes del dosel se fraccionan en divisiones cenital y azimutal (sectores de dosel). La fracción de cielo (coeficiente de transmisión del haz solar) visible en cada división se analiza contando la porción de cielo de los píxeles de la imagen. Entonces, el equipo captura imágenes de amplio ángulo del dosel mientras estima el IAF y mide los niveles de radiación fotosintéticamente activa (PAR) por sitio de muestreo. Las imágenes se actualizan en vivo en el monitor incorporado, proporcionando datos instantáneos para verificación y análisis con el software integrado. El IAF se representa por valores que varían de 0 a 10, donde 0 equivale a un área sin dosel o suelo desnudo y 10 representa un dosel denso (Bio-Science, 2016). Las condiciones óptimas del cielo para las mediciones deben ser bajo una cubierta uniforme de nubes durante la mañana o al final de la tarde (cantidad de radiación baja) (Bio-Science, 2016).

\subsection{Medición de precipitación efectiva}

Para medir la PE se instalaron 9 pluviógrafos automáticos de resolución de $0,2 \mathrm{~mm}$ a una altura sobre el suelo de 1,20 m. Los pluviógrafos fueron calibrados in situ y sobre cada uno se colocó una malla plástica para recoger la hojarasca y así evitar su taponamiento. La descarga de datos, mantenimiento y limpieza de los equipos instalados se realizó semanalmente a partir del 9 de Marzo de 2019 hasta el 8 de Marzo de 2020.

Los registros fueron agregados para tener una base de datos con frecuencia de 5 minutos. La cantidad de PE obtenida corresponde a la acumulación diaria y anual de los valores registrados por los pluviógrafos en cada punto de muestreo dentro del bosque. En caso de pérdida de datos por falla en la descarga o por taponamiento, se realizó un relleno de datos diarios mediante el método de regresión lineal de los valores del pluviógrafo que presentó pérdida de datos con el pluviógrafo que mostró la mejor correlación. 


\subsection{Relación entre DD, IAF y PE.}

Primero se comparó la DD con el IAF mediante correlación por el método de Pearson, con el objeto de conocer si estas dos variables muestran la mis- ma información con respecto a la cobertura del dosel. Luego se realizaron correlaciones y regresiones lineales de la DD y el IAF con la PE para conocer qué variable permite identificar la variabilidad de la PE referente al dosel del bosque.

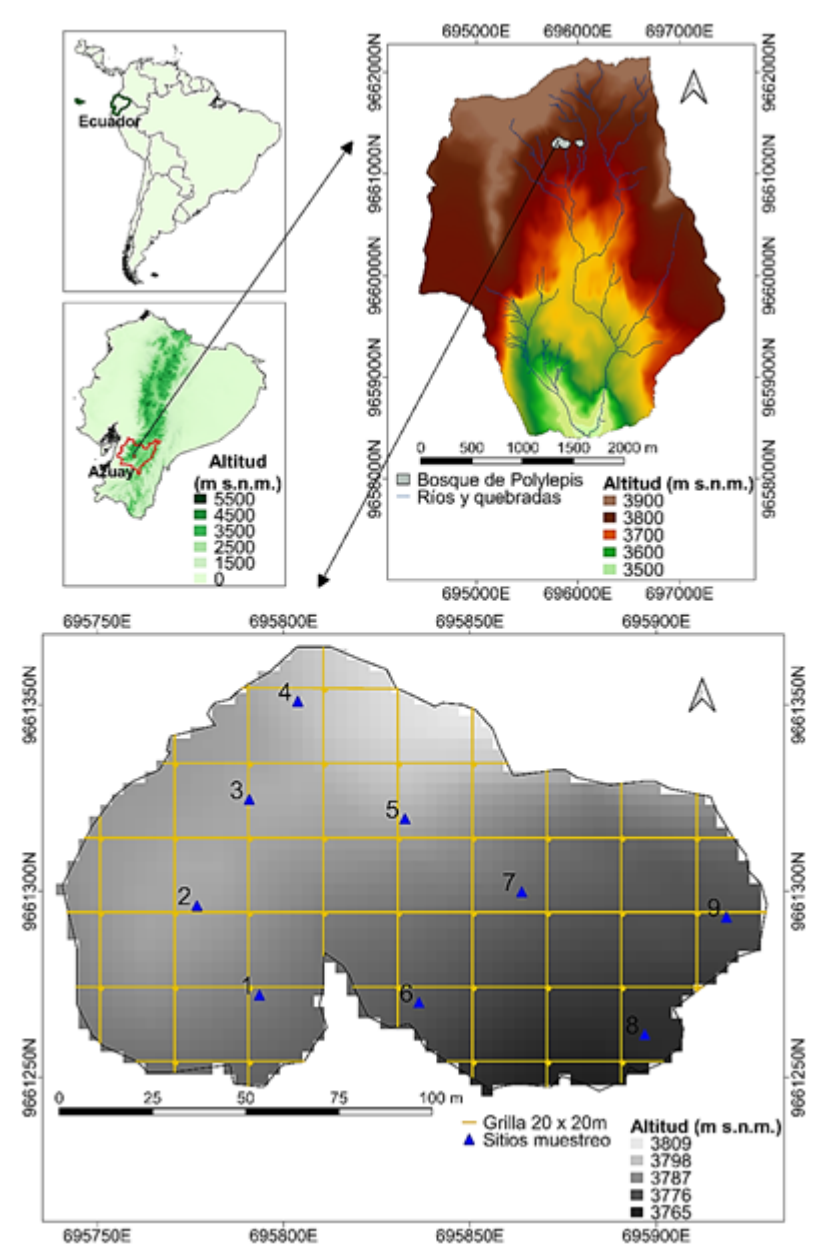

Figura 1. Área de estudio localizada en el Observatorio Ecohidrológico Zhurucay al Sur de Ecuador.

\section{Resultados}

\subsection{Densidad del dosel}

La variabilidad espacial del dosel modelada demostró mejor ajuste y fidelidad con las características del terreno al emplear interpolación por el método de Kriging que con respecto a IDW y a Polígonos de Thiessen (37 puntos, grilla $20 \times 20 \mathrm{~m}$ ). Tal método se ajustó de mejor manera a los datos, presentando los errores más bajos.
En la Figura 2 se observa que la DD del bosque de Polylepis presenta un rango de variación espacial entre $62,5 \%$ hasta $95,2 \%$. En ciertas zonas predominan valores entre 87 a $91 \%$, que representan aproximadamente un área de $550 \mathrm{~m}^{2}$.

Como se indicó en la sección 3.4, se consideraron 9 sitios de muestreo para PE, donde se midió la DD e IAF (Figura 2). En la Figura 3 se observa que el porcentaje de DD en los 9 sitios varía en un rango 
de $79 \%$ (sitio 1) a $96 \%$ (sitio 5). El valor promedio del porcentaje de DD fue de $88 \pm 5,8 \%$. El coeficien- te de variación resultó bajo, sin superar el 10\%, lo cual indica homogeneidad en los datos.

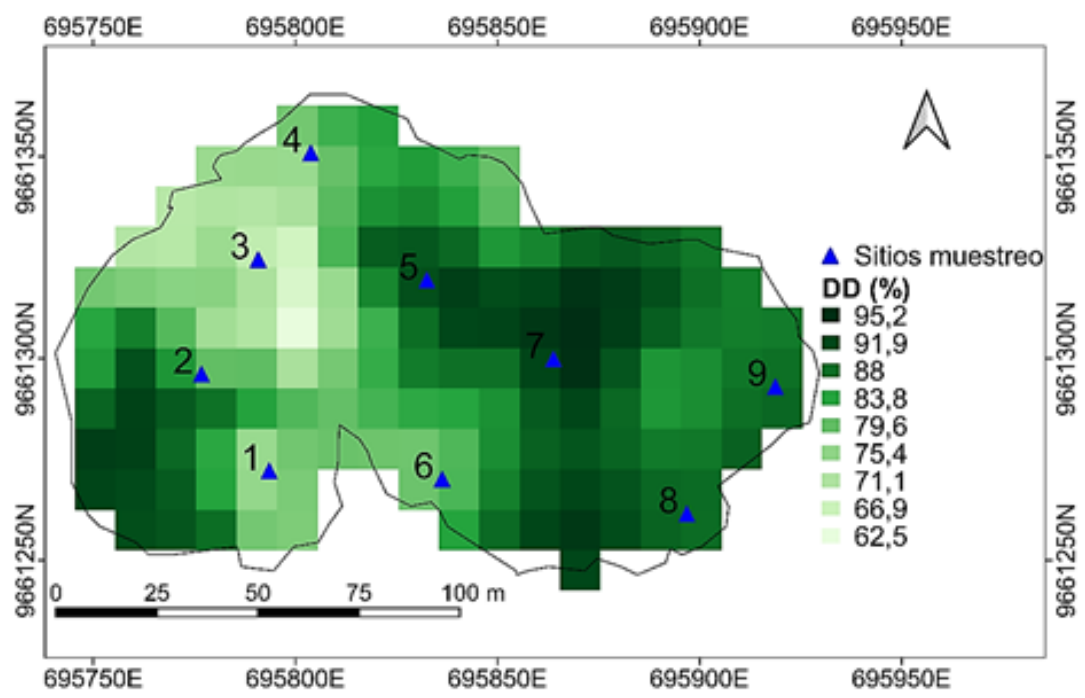

Figura 2. Variabilidad espacial de la densidad del dosel por el método de interpolación Kriging y sitios de muestreo de precipitación efectiva.

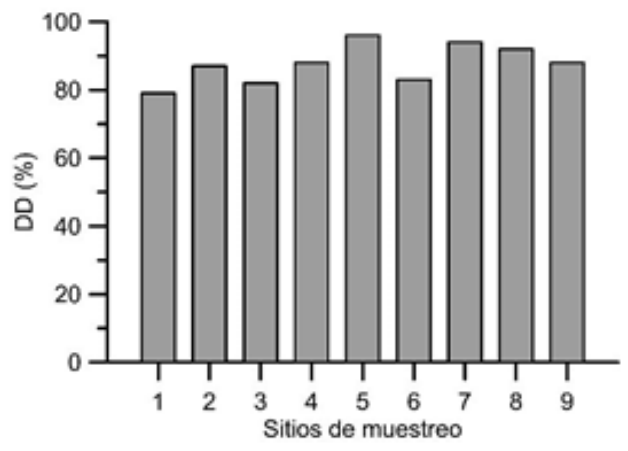

Figura 3. Porcentaje de densidad de dosel.

\section{2 Índice de área foliar}

Tal como se muestra en la Figura 4 el IAF estimado en los 9 sitios de muestreo varía entre $2,05 \mathrm{~m}^{2} \mathrm{~m}^{-2}$ (sitio 1) y $2,79 \mathrm{~m}^{2} \mathrm{~m}^{-2}$ (sitio 5), con un promedio de $2,43 \pm 0,25 \mathrm{~m}^{2} \mathrm{~m}^{-2}$. De manera análoga con la DD, el coeficiente de variación fue de $10 \%$, confirmando una baja variabilidad de IAF dentro del bosque.

\subsection{Precipitación efectiva}

Se encontró que el pluviógrafo del sitio 2 presentó el mayor porcentaje de datos perdidos de PE con un 3,6\% de datos a rellenar; cabe recalcar que este procedimiento no afectó los resultados debido a que no sobrepasa el límite aceptable de valores perdidos $(10 \%)$.

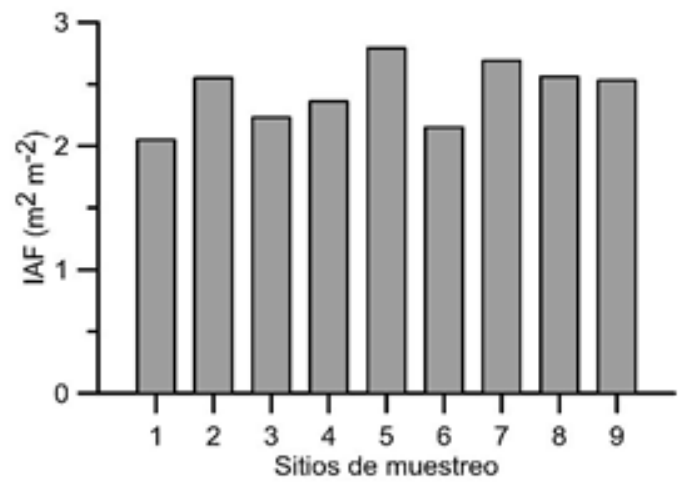

Figura 4. Índice de área foliar.

La PE media anual estimada mediante pluviógrafos en 9 sitios del bosque de Polylepis fue de 773,2 $\mathrm{mm}$ año ${ }^{-1} \pm 212,6$ con una media diaria de $2,1 \pm$ $0,58 \mathrm{~mm} \mathrm{día}^{-1}$ (Tabla 1). La cantidad anual de PE varía entre 484,9 a $1191,6 \mathrm{~mm}^{2}$ ano $^{-1}$, y la cantidad media diaria anual presentó una variación de 1,3 a $3,3 \mathrm{~mm} \mathrm{día}^{-1}$, valores que corresponden al sitio 9 y 
1 respectivamente (Tabla 1 y Figura 5).

El coeficiente de variación tanto para valores acumulados anual como para valores de media diaria anual de todos los sitios representa el $28 \%$ de variabilidad. Al analizar la PE diaria anual en cada sitio de muestreo se observa que los datos reportan un CV de $160 \%$ (sitio 1) hasta 180\% (sitios 2, 5 y 8), lo que indica que la PE en el bosque en estudio presenta alta heterogeneidad.

En la Figura 5 se observa que los diagramas de caja para los sitios de estudio presentan valores atípicos que se concentran sobre el límite superior, posiblemente como respuesta a eventos particulares de precipitación, que en este caso son eventos diarios que superan los $10 \mathrm{~mm}$.

Tabla 1. Precipitación efectiva (PE) acumulada anual, media diaria anual, desviación estándar $(\sigma)$ diaria anual y coeficiente de variación $(\mathrm{CV})$ diario anual.

\begin{tabular}{ccccc}
\hline $\begin{array}{c}\text { Sitio de } \\
\text { muestreo }\end{array}$ & $\begin{array}{c}\text { PE anual }(\mathbf{m m} \\
\left.\text { año }^{-1}\right)\end{array}$ & $\begin{array}{c}\text { PE media diaria } \\
\text { anual }\left(\mathbf{m m} \text { día }^{-1}\right)\end{array}$ & $\begin{array}{c}\sigma \text { diaria } \\
\text { anual }\end{array}$ & $\begin{array}{c}\text { CV } \\
\text { diario anual } \\
(\%)\end{array}$ \\
\hline 1 & 1191,6 & 3,3 & 5,2 & 160 \\
2 & 825,4 & 2,3 & 4,1 & 180 \\
3 & 694,5 & 1,9 & 3,2 & 170 \\
4 & 743,0 & 2,0 & 3,5 & 170 \\
5 & 592,7 & 1,6 & 2,9 & 180 \\
6 & 862,5 & 2,4 & 4,1 & 170 \\
7 & 944,9 & 2,6 & 4,7 & 180 \\
8 & 619,1 & 1,7 & 2,9 & 170 \\
9 & 484,9 & 1,3 & 2,3 & 170 \\
\hline $\bar{x}$ & 773,2 & 2,1 & & \\
$\sigma$ & 212,6 & 0,58 & 3,8 & \\
$\mathbf{C V}$ & 28 & 28 & & 180 \\
\hline
\end{tabular}

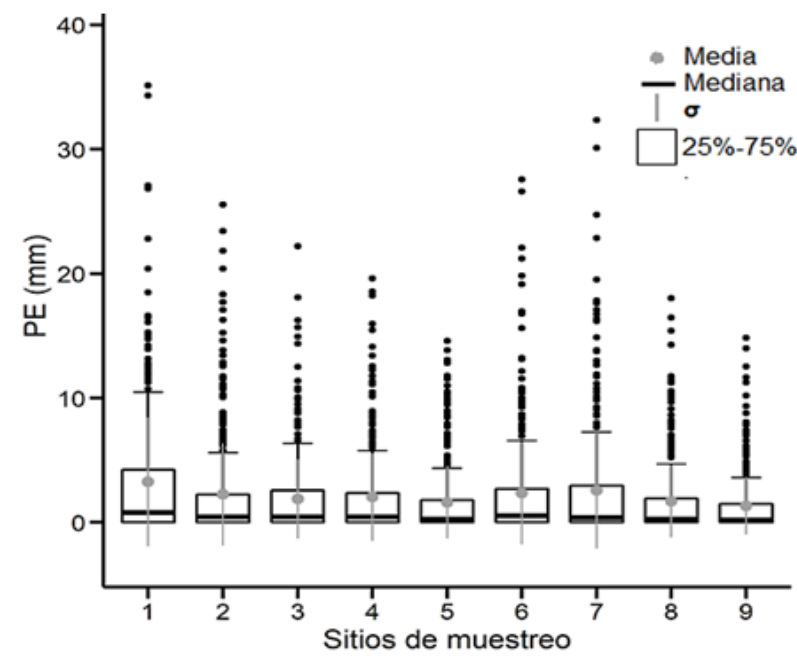

Figura 5. Precipitación efectiva diaria. 


\subsection{Relación entre DD, IAF y PE}

Como se esperaba, la correlación entre el IAF y la DD es altamente significativa con un valor $\mathrm{p}<0,05$ y un coeficiente $R^{2}$ de 0,913 . En la Figura 6 se observa que la DD tiende a incrementar con el aumento del IAF.

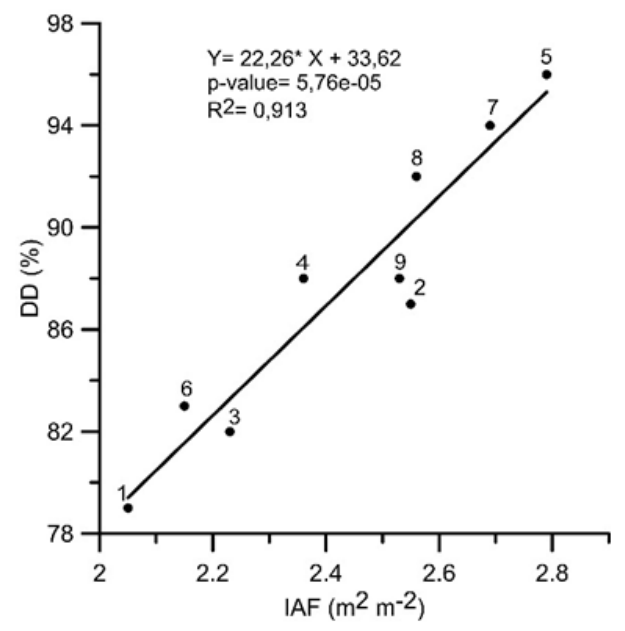

Figura 6. Relación entre índice de área foliar (IAF) y densidad del dosel (DD) (1-9 sitios de muestreo).

Los coeficientes de correlación indican la existencia de una relación inversa entre las variables de cobertura vegetal (IAF y DD) con la PE, reportando valores de $-0,535$ y $-0,524$ respectivamente; como se esperaba, la PE tiende a disminuir con el incremento de la DD o IAF (Figura 7). Sin embargo, los valores $p>0,05$ indican que la relación resulta ser no significativa. Al aplicar el método de regresión lineal se encontró coeficientes $R^{2}$ bajos de 0,286 y 0,275 que indican la poca dependencia o respuesta de la PE a estas variables de cobertura del dosel.
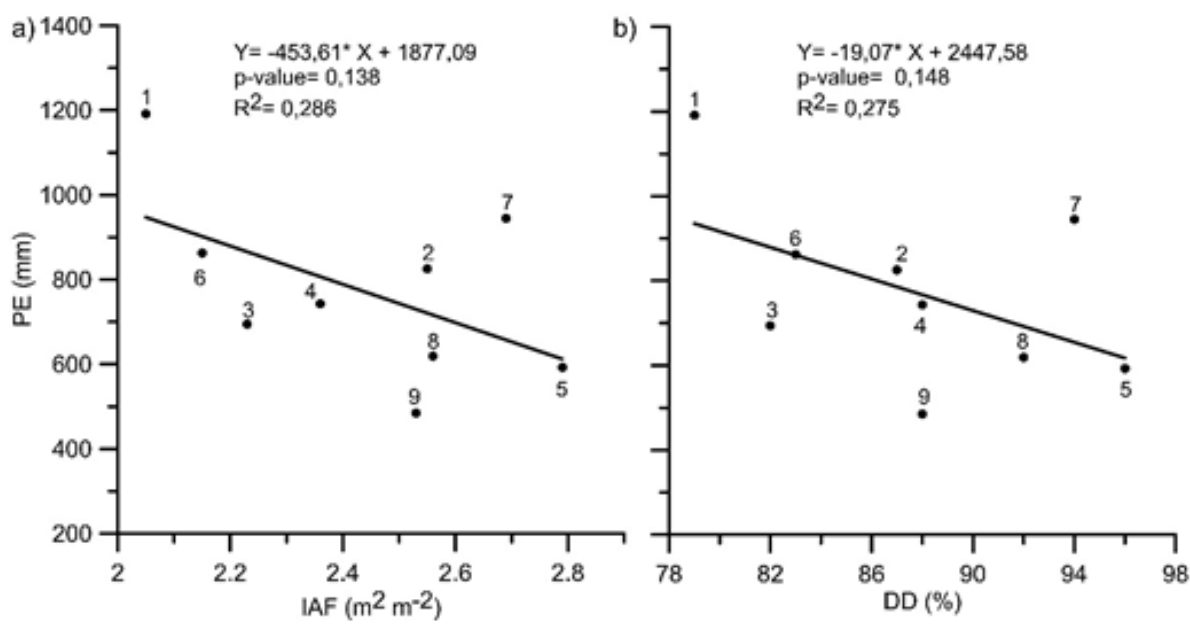

Figura 7. Relación entre a) índice de área foliar (IAF), b) densidad del dosel (DD) con la precipitación efectiva (PE) (1-9 sitios de muestreo). 


\section{Discusión}

La información sobre el IAF, DD, PE y la relación existente entre tales variables para el bosque de Polylepis reticulata analizado, se convierte en información importante para los bosques altoandinos; facilita la comprensión y sienta una línea base sobre el rol que tiene la vegetación en procesos hidrológicos que suceden en bosques de páramo.

\subsection{Caracterización de la cobertura del do- sel}

Múltiples investigaciones realizadas en bosques de ecosistemas andinos revelan valores de IAF y DD (Tabla 2). Tales estudios también caracterizan la cobertura del dosel y la relación con diferentes funciones ecológicas.

Tabla 2. IAF y DD de bosques localizados en ecosistemas andinos.

\begin{tabular}{|c|c|c|c|c|}
\hline $\begin{array}{l}\text { Ecosistema y tipo } \\
\text { de bosque }\end{array}$ & $\begin{array}{c}\text { Sitio de estudio y } \\
\text { elevación } \\
\text { (m s.n.m.) }\end{array}$ & $\begin{array}{l}\text { Medición IAF } \\
\left(\mathbf{m}^{2} \mathbf{m}^{-2}\right)\end{array}$ & $\begin{array}{c}\text { Medición DD } \\
(\%)\end{array}$ & Referencia \\
\hline $\begin{array}{l}\text { Páramo: Bosque de } \\
\text { Polylepis reticulata }\end{array}$ & $\begin{array}{c}\text { Ecuador, } \\
\text { Cuenca del Río } \\
\text { Zhurucay, } \\
(3765-3809)\end{array}$ & $2,05-2,79$ & $79-96$ & $\begin{array}{l}\text { Presente } \\
\text { estudio }\end{array}$ \\
\hline $\begin{array}{c}\text { Páramo: Bosque } \\
\text { Polylepis reticulata }\end{array}$ & $\begin{array}{c}\text { Ecuador, } \\
\text { Parque Nacional } \\
\text { Cajas, } \\
(3735-3930)\end{array}$ & $2,60-6,17$ & & Pinos y col. (2017) \\
\hline $\begin{array}{c}\text { Páramo: Bosque de } \\
\text { Polylepis pauta } \\
\text { e incana }\end{array}$ & $\begin{array}{c}\text { Ecuador, } \\
\text { Papallacta, } \\
(3500-4100)\end{array}$ & & $46,7-75$ & Cierjacks y col. (2007) \\
\hline $\begin{array}{l}\text { Páramo: Bosque de } \\
\text { Polylepis australis }\end{array}$ & $\begin{array}{c}\text { Argentina, } \\
\text { Córdoba, } \\
(1400-2500) \\
\end{array}$ & & $8-72$ & Renison, Hensen y Suarez (2011) \\
\hline $\begin{array}{l}\text { Páramo: Plantación } \\
\text { de pino }\end{array}$ & $\begin{array}{c}\text { Ecuador, } \\
\text { Azuay, } \\
(3500-3700) \\
\end{array}$ & $0,23-2,22$ & $5,5-74,7$ & Alvarado y Muñoz (2017) \\
\hline $\begin{array}{c}\text { Páramo: Plantación } \\
\text { de pino }\end{array}$ & $\begin{array}{c}\text { Ecuador, } \\
\text { Azuay, } \\
(3600-3800)\end{array}$ & & $19,3-64,8$ & Quiroz y col. (2019) \\
\hline $\begin{array}{c}\text { Páramo: Plantación } \\
\text { de pino } \\
\text { Bosque montano } \\
\text { alto }\end{array}$ & $\begin{array}{c}\text { Ecuador, } \\
\text { Azuay, } \\
(3800 \text { y } 2500)\end{array}$ & 5,5 y 0,2 & & Jadán y col. (2019) \\
\hline $\begin{array}{c}\text { Bosques } \\
\text { siempreverdes } \\
\text { montano alto y bajo }\end{array}$ & $\begin{array}{c}\text { Ecuador, } \\
\text { Azuay, } \\
(2000-3800) \\
\end{array}$ & $1,6-2,5$ & $53-72$ & Alvarado y Cobos (2019) \\
\hline $\begin{array}{l}\text { Bosque montano } \\
\text { bajo }\end{array}$ & $\begin{array}{c}\text { Ecuador, } \\
\text { Loja - Zamora, } \\
(1900-2000)\end{array}$ & $5,2-9,3$ & & Fleischbein y col. (2005) \\
\hline $\begin{array}{l}\text { Bosque montano } \\
\text { tropical }\end{array}$ & $\begin{array}{c}\text { Ecuador, } \\
\text { Loja, } \\
(1050-3060) \\
\end{array}$ & $5,1-2,9$ & & Moser, Hertel y Leuschner (2007) \\
\hline $\begin{array}{l}\text { Bosque montano } \\
\text { tropical nublado }\end{array}$ & $\begin{array}{c}\text { Perú, } \\
\text { Parque Nacional } \\
\text { Yanachaga- } \\
\text { Chemillén, } \\
(2815 \text { - 2468) }\end{array}$ & $2,5-2,9$ & $87,9-90,7$ & Gomez y col. (2008) \\
\hline
\end{tabular}




\subsubsection{Estudios de índice de área foliar}

Los valores de IAF del bosque de Polylepis estudiado se encuentran dentro de los valores inferiores reportados por Pinos y col. (2017) para bosques de Polylepis reticulata en el Parque Nacional Cajas, que presentan un IAF entre 2,60 a $6,17 \mathrm{~m}^{2} \mathrm{~m}^{-2}$ (media de $3,96 \mathrm{~m}^{2} \mathrm{~m}^{-2}$ ). Esta diferencia puede deberse al método utilizado para la determinación del IAF, ya que el autor empleó área foliar específica y densidad de árboles por parcela de estudio. En estudios realizados en plantaciones de pino establecidas en ecosistemas de páramo, Alvarado y Muñoz (2017) reportan un rango de IAF de 0,23 a $2,22 \mathrm{~m}^{2} \mathrm{~m}^{-2}$ (media de $0,92 \mathrm{~m}^{2} \mathrm{~m}^{-2}$ ), valores que resultan ser menores a los encontrados en este estudio, posiblemente debido a la forma de las hojas y densidad de la plantación. Así también, Jadán y col. (2019) registraron valores muy bajos de $\operatorname{IAF}\left(0,2 \mathrm{~m}^{2} \mathrm{~m}^{-2}\right)$ en plantaciones de similar especie y un IAF de $5,5 \mathrm{~m}^{2}$ $\mathrm{m}^{-2}$ en bosques montano alto, que resulta mayor a lo encontrado. En un estudio realizado en bosques siempreverdes montano alto y bajo localizados al sur de Ecuador, Alvarado y Cobos (2019) reportan resultados de IAF con una variabilidad menor a la del presente estudio. Fleischbein y col. (2005) indican que un bosque montano bajo al sur de Ecuador presenta un IAF entre 5,2 y 9,3 $\mathrm{m}^{2} \mathrm{~m}^{-2}$ (media de $7,3 \mathrm{~m}^{2} \mathrm{~m}^{-2}$ ), valores más altos que los identificados en el presente estudio. Moser, Hertel y Leuschner (2007) en bosques montano tropical al sur de Ecuador, indican que al incrementar la altitud el tamaño de las hojas es menor; por consiguiente, el IAF disminuye. Gomez y col. (2008) en bosques montano tropical del Perú, encontraron que el IAF es de 2,5 $\pm 0,7$ a $2,9 \pm 0,2 \mathrm{~m}^{2} \mathrm{~m}^{-2}$, valores que resultan ser mayores a los encontrados en el bosque de Polylepis.

\subsubsection{Estudios de densidad del dosel}

Estudios realizados en Polylepis han determinado un porcentaje de DD; como es el caso del estudio desarrollado por Cierjacks y col. (2007) en bosques de Polylepis pauta e incana, en los que se encontró valores de $46,7 \%$ cerca del borde y de $65-75 \%$ en la parte interna del bosque, datos que resultan ser menores a los de nuestro estudio tanto cerca del borde $(79,83$ y $88 \%$; sitios 1,6 y 9) como en la parte interna del bosque (94 - $96 \%$; sitio 7 y 5). De igual manera, Renison, Hensen y Suarez (2011) en un bosque de Polylepis australis identificaron que la variabilidad de DD es menor a la encontrada en el presente estudio con porcentajes de 8, 23, 54 y hasta un $72 \%$. Esto puede deberse a que los resultados de los estudios mencionados presentan un sesgo de subjetividad que los obtenidos en nuestro estudio, debido a que fueron determinados de forma visual, método que depende completamente de la experiencia del técnico. En plantaciones de pino ubicadas en ecosistemas de páramo, Alvarado y Muñoz (2017) reportan que estos bosques presentan un rango de DD de 5,5 a 74,7\% (media de 44,5\%). Al igual, Quiroz y col. (2019) en su investigación presenta porcentajes de DD (19,3\% - 64,8\%) menores a los encontrados en el sitio de estudio. En bosques siempreverdes montano alto y bajo localizados al sur de Ecuador, se registraron promedios de DD de $53 \pm 4 \%$ y $72 \pm$ $3,2 \%$ valores más bajos a los encontrados en el presente estudio (Alvarado y Cobos, 2019). El estudio realizado por Gomez y col. (2008), en un bosque montano tropical del Perú presenta similitudes en los valores de DD $(87,9 \% \pm 6,2$ a $90,7 \% \pm 1,6)$ con los datos reportados en el bosque de Polylepis.

De acuerdo a lo observado, la poca similitud tanto del IAF como del porcentaje de DD entre bosques, se debe a que tales variables dependen de condiciones propias del sitio, las que generalmente influyen sobre el desarrollo y características de cada especie arbórea. Parámetros influyentes son: El tamaño de hoja, densidad de árboles por unidad de superficie, arquitectura y estructura de ramas y la topografía; por lo que pueden variar significativamente de un sitio a otro, así se trate de la misma especie forestal.

La estrecha relación entre IAF y DD queda evidenciada mediante análisis de regresión, revelando una fuerte proporcionalidad entre ambas variables, lo que concuerda con el estudio realizado por Buckley, Isebrands y Sharik (1999), en el que reportan un $\mathrm{R}^{2}$ de 0,93 y 0,99 en bosques de roble y pino, explicando que esto es debido a que los bosques de estudio presentan una estructura uniforme. Sin embargo, este autor también comenta que la relación entre las variables puede cambiar cuando existe diferencias entre las especies forestales, arquitectura de la copa y etapa de desarrollo. 


\subsection{Variabilidad de la precipitación efecti- va y relación con la cobertura del dosel}

En este estudio se evidenció que la PE dentro del bosque de Polylepis fue heterogénea. La literatura no reporta otro estudio de PE realizado en bosques de Polylepis. Estudios realizados en bosques tropicales premontano (Teale y col., 2014), bosque montano bajo (Fleischbein y col., 2005) y en un bosque mixto de roble (Staelens y col., 2006), también encontraron que la PE es muy variable. Estos estudios dan una posible explicación de que esta variable se encuentra influenciada por la arquitectura del dosel, características morfológicas de las hojas y en algunos casos por una mayor carga de epífitas sobre el dosel que pueden generar más puntos de goteo, lo que conlleva a una mayor variabilidad de PE. De igual manera el estudio realizado por Zimmermann, Wilcke y Elsenbeer (2007) explica que la variabilidad espacial depende principalmente de la complejidad del dosel y es influenciada por el número de especies por área, altura irregular, presencia de epífitas, edad, estructura y disposición de los árboles. Otra posible explicación es que la PE también depende de la profundidad de los eventos de precipitación, razón por la que la variabilidad espacial de PE incrementa, sugiriendo que los patrones espaciales del volumen de PE pueden ser independientes del ecosistema. Germer, Elsenbeer y Moraes (2006) y Roth, Slatton y Cohen (2007) muestran que existe características como la diversidad en las especies, el tamaño y la estructura de la vegetación que dan como resultado la distribución de la lluvia, los puntos de goteo y almacenaje localizado en el dosel inferior produciendo patrones espacialmente heterogéneos. Algo similar muestran Zimmermann, Zimmermann y Elsenbeer (2009) y Macinnis y col. (2014) en sus estudios, pues es la vegetación la que influye en el movimiento del agua a través del dosel, ya que ciertas formas o su distribución dentro del área forestal pueden crear puntos de goteo. Además, otros estudios como el de Zimmermann y col. (2008) indican que la PE se ve afectada por las condiciones antecedentes del dosel como es la humedad. En nuestro estudio, el coeficiente de variación diario anual resulta ser mayor que el acumulado anual. Carlyle y Price (2007) explican que cuando la PE se observa en una resolución temporal de menor agregación, como es el caso de una agregación diaria o por eventos, este valor se encuentra influenciado por las condiciones de intensidad de la precipitación bruta, e incluso llega a depender de las condiciones del viento. El coeficiente de variación de la PE puede incrementar o disminuir cuando la intensidad de la lluvia es su principal factor de cambio (Weiqing y col., 2007).

A pesar que en el presente estudio las correlaciones encontradas entre las variables IAF, DD y la PE no se muestran estadísticamente significativas, los resultados evidencian una proporcionalidad inversa entre las variables de cobertura del dosel y la $\mathrm{PE}$, lo que concuerda con previas investigaciones en las que reportan que, a medida que aumenta IAF, la PE tiende a disminuir (Llorens y Gallart, 2000; Loescher, Powers y Oberbauer, 2002; Nadkarni y Sumera, 2004). Del mismo modo, Holwerda, Scatena y Bruijnzeel (2006) indican que la PE en un bosque de Puerto Rico fue mayor en zonas con bajas cantidades de dosel, debido a que una superficie de dosel más pequeña se correlaciona con una menor cantidad de agua interceptada. En el estudio realizado por Fleischbein y col. (2005) se reporta una correlación negativa entre IAF y PE (Pearson $r$ $=-0.49$ ) coeficiente ligeramente menor al encontrado en nuestro trabajo. Sin embargo, al comparar con la intercepción, Fleischbein y col. (2005) muestra que el IAF solamente explica el $12 \%$ de la variación, atribuyendo a que el área de cobertura vegetal medida sobre los colectores de PE es mayor al área del colector. De este modo, la diferencia de área de captura de los medidores de PE y el área cubierta por los equipos de IAF y DD podrían explicar la variabilidad de la intercepción, o en nuestro caso la variación de PE en valores similares de IAF y los bajos coeficientes de determinación entre las variables de cobertura de dosel con la PE. La comparación de los resultados con el estudio de Teale y col. (2014) en un bosque de Costa Rica, confirma que la relación entre IAF y PE es estadísticamente no significativa, probablemente debido a que las localidades con similar IAF pueden tener diferente configuración del tipo de hoja, cobertura de madera, orientación del follaje y ramas entre otras características que forman puntos de retención y puntos de goteo. Por tal razón, es claro que la vegetación influye en la forma en que el agua se mueve a través del dosel; si bien la PE generalmente es menor que la precipitación bruta, ciertos arreglos y formas de vegetación pueden crear puntos de goteo logrando exceder en gran medida a la precipitación. En general, los resultados de los estudios que intentaron relacionar 
la cobertura del dosel o las características de la vegetación con la PE han sido débiles (Keim, Skaugset y Weiler, 2005). De igual manera lo indica Zimmermann, Zimmermann y Elsenbeer (2009), al hablar de la relación entre la apertura del dosel y la PE en los puntos de medición, donde se observa más débil a medida que aumenta la magnitud del evento de precipitación. En un estudio realizado por Molina y col. (2019) en bosques de pino y roble, indica que la PE no presenta una relación significativa cuando los valores de DD son menores al $60 \%$. Sin embargo, al incrementar esta variable en un rango de 60 a $100 \%$ se observa un patrón significativo de disminución de la PE con el incremento de la DD, mostrando una correlación negativa de 0,51 y 0,61 respectivamente. Autores como Park y Cameron (2008) encontraron que existe una interacción entre los impactos que producen las características del dosel en la PE con la influencia de las características del evento de precipitación; sin embargo, mediante el análisis estadístico no se pudo identificar ningún patrón. (Levia y col., 2011) indica que los patrones espaciales de la PE varían notablemente entre ecosistemas por lo que no es posible identificar una relación entre el dosel y la PE.

Como anteriormente ha sido mencionado por varios autores, la posible explicación a la relación no significativa entre las variables de cobertura del dosel y la PE, es que este proceso hidrológico no depende solo de la DD o IAF, ya que existen otros factores tanto de la vegetación (estructura, arquitectura de las ramas, densidad, edad, ángulo de inclinación de las hojas) como climáticos (intensidad de la lluvia y viento).

Por otro lado, estudios realizados en bosques deciduos resaltan el efecto de la dinámica del follaje sobre la PE, debido a que presentan períodos notables de pérdida de hojas, siendo lo contrario en bosques perennifolios como es el caso de Polylepis. Sin embargo, es claro que su follaje presenta una dinámica que consiste en desfronde y renovación de las hojas, como lo indica Pinos (2014), reportando que bosques de Polylepis presentan tasas de desfronde de 0,61 año ${ }^{-1}$ y el período de renovación foliar se da en 1,75 años. Por esta razón, esta dinámica debería ser tomada en cuenta para futuras investigaciones, con un mayor número de puntos de medición de cobertura del dosel, en diferentes épocas o temporadas del año.

\section{Conclusiones}

Este estudio es pionero en comparar mediciones de IAF y DD para su relación con la PE en bosques de Polylepis, que se caracterizan por ser parte de ecosistemas de páramo. El IAF y DD son variables que difieren de acuerdo a las condiciones a las que se encuentran expuestas las especies forestales durante su crecimiento, por ejemplo, nutrientes en el suelo, agua, viento, precipitación, temperatura. Así como de características propias de las especies que conforman el bosque como: forma y tamaño de la hoja, arquitectura y estructura de la ramificación, altura, edad, entre otras.

Se encontró una fuerte relación entre las variables de dosel medidas, que a su vez proporcionan similar información respecto a la cobertura, con lo cual se concluye que cualquiera de las dos técnicas podría ser utilizada para estimar la cobertura del dosel. Sin embargo, por su mayor comodidad, facilidad de operación y principalmente bajo costo, la técnica de medición del porcentaje de DD mediante el método del densiómetro esférico, resulta la más óptima para esta actividad.

Al estudiar la precipitación efectiva, uno de los principales procesos hidrológicos que se produce a nivel del dosel, se pudo constatar que no solo está influenciada por las variables antes mencionadas, pues es el conjunto de características y distribución de las especies forestales, lo que incrementa la complejidad vegetal dentro del bosque y a su vez la heterogeneidad de la PE. Además, mientras más fina es la resolución temporal usada en la estimación de la PE (diario, horario, minutos), posiblemente está influenciada por condiciones ambientales como son la intensidad y duración de la precipitación, viento, radiación solar, condiciones antecedentes sean secas o de humedad del dosel.

La relación no significativa entre IAF y DD con la PE, se puede atribuir a la diferencia de áreas de medición de las 2 variables (cobertura del dosel y precipitación), ya que el área que abarcan los colectores de lluvia, en este caso los pluviógrafos, es mucho menor al área proyectada por los equipos utilizados para medir la cobertura del dosel. Por lo tanto, las mediciones de la cobertura vegetal - con cualquiera de las dos técnicas - resultan limitadas para caracterizar apropiadamente la variabilidad de 
la PE en el ecosistema estudiado.

\section{Agradecimientos}

El presente estudio es el resultado de la Maestría en Hidrología con mención en Ecohidrología del Departamento de Recursos Hídricos de la Universidad de Cuenca. Este estudio se ejecutó en el marco del proyecto "The Ecohydrologic functions of Polylepis forests within the Andean páramo and their resilience and their vulnerability to climate change", financiado por la Dirección de Investigación de la Universidad de Cuenca (DIUC) y la Universidad de New Hampshire. Un agradecimiento al Ing. Franklín Marín y Blgo. Aldemar Carabajo por la ayuda y apoyo brindado en las campañas de campo. Al Ing. Oswaldo Jadán de la Facultad de Ciencias Agropecuarias de la Universidad de Cuenca por la colaboración con los equipos para medir la cobertura del dosel.

\section{Referencias}

Alfaro, G. (2015). «Caracterización de la infiltración en bosques de Polylepis spp., de 11 y 29 años, Parque Nacional Huascarán, Quebrada Quilcayhuanca, Huaraz, Ancash». Tesis de grado. Perú: Universidad Agraria La Molina. Online: https: //bit.ly/3ooQaZh.

Alvarado, A. y L. Muñoz (2017). «Evaluación de la regeneración natural y su relación con la altitud y cobertura de dosel en plantaciones no manejadas de Pinus patula en zonas alto Andinas, en la provincia del Azuay». Tesis de grado. Cuenca, Ecuador: Universidad de Cuenca. Online: https: //bit.ly/3ol1YvI.

Alvarado, I. y C. Cobos (2019). «Relaciones entre la estructura y cobertura arbórea con el carbono almacenado en bosques montanos Andinos en el macizo del Cajas, Azuay-Ecuador». Tesis de grado. Cuenca, Ecuador: Universidad de Cuenca. Online: https://bit.ly/3yc33dL.

Berger, T. y col. (2008). "Throughfall fluxes in a secondary spruce (Picea abies), a beech (Fagus sylvatica) and a mixed spruce-beech stand». En: Forest Ecology and Management 255.3-4, 605-618. Online: https://bit.ly/3ftBtQD.

Bio-Science (2016). Manual CI-110 / 120 Plant Canopy Imager.
Brauman, K., D. Freyberg y G. Daily (2010). «Forest structure influences on rainfall partitioning and cloud interception: A comparison of native forest sites in Kona, Hawai'i». En: Agricultural and Forest Meteorology 150.2, 265-275. Online: https: //bit.ly/3ot1jrY.

Buckley, D., J. Isebrands y T. Sharik (1999). «Practical field methods of estimating canopy cover, PAR, and LAI in Michigan oak and pine stands». En: Northern Journal of Applied Forestry 16.1, 25-32. Online: https:/ / bit.ly/3brumXW.

Carlyle, D. y A. Price (2007). «Modelling canopy interception loss from a Madrean pine-oak stand, northeastern Mexico». En: Hydrological Processes: An International Journal 21.19, 2572-2580. Online: https:/ / bit.ly/2Rdx7Fr.

Carrillo, G. y col. (2019). «The breathing of the Andean highlands: Net ecosystem exchange and evapotranspiration over the páramo of southern Ecuador». En: Agricultural and Forest Meteorology 265, 30-47. Online: https:/ / bit.ly/2SS1odg.

Cierjacks, A. y col. (2007). "Impact of sowing, canopy cover and litter on seedling dynamics of two Polylepis species at upper tree lines in central Ecuador». En: Journal of Tropical Ecology, 309-318. Online: https://bit.ly/3hwP78i.

Cook, J. y col. (1995). «Spherical densiometers produce biased estimates of forest canopy cover». En: Wildlife Society Bulletin 23.4, 711-717. Online: https://bit.ly/3uTrieF.

Córdova, M., G. Carrillo y R. Célleri (2013). «Errores en la estimación de la evapotranspiración de referencia de una zona de Páramo Andino debidos al uso de datos mensuales, diarios y horarios.» En: Aqua-LAC 5.2, 14-22. Online: https: //bit.ly/3fem8mM.

Córdova, M. y col. (2015). «Evaluation of the Penman-Monteith (FAO 56 PM) method for calculating reference evapotranspiration using limited data». En: Mountain Research and Development 35.3, 230-239. Online: https: / / bit.ly / 3 bplNwH.

Crockford, R. y D. Richardson (2000). «Partitioning of rainfall into throughfall, stemflow and interception: effect of forest type, ground cover and climate». En: Hydrological processes 14.16-17, 2903-2920. Online: https:/ / bit.ly/3buU0ei.

Domic, A., G. Camilo y J. Capriles (2014). «Smallscale Farming and Grazing Reduce Regeneration of Polylepis tomentella (Rosaceae) in the Se- 
miarid Andes of Bolivia». En: Biotropica 46.1, 106-113. Online: https://bit.ly/33INigu.

Fleischbein, K. y col. (2005). «Rainfall interception in a lower montane forest in Ecuador: effects of canopy properties». En: Hydrological Processes: An International Journal 19.7, 1355-1371. Online: https:/ / bit.ly/3eMJGjI.

Gareca, E. y col. (2010). «Polylepis woodland remnants as biodiversity islands in the Bolivian high Andes». En: Biodiversity and conservation 19.12, 3327-3346. Online: https:/ / bit.ly/3osFHvW.

Germer, S., H. Elsenbeer y J. Moraes (2006). «Throughfall and temporal trends of rainfall redistribution in an open tropical rainforest, southwestern Amazonia (Rondônia, Brazil)». En: $\mathrm{Hy}$ drology and Earth System Sciences 10.3, 383-393. Online: https://bit.ly/3oie8FJ.

Gerrits, A., L. Pfister y H. Savenije (2010). «Spatial and temporal variability of canopy and forest floor interception in a beech forest». En: Hydrological Processes 24.21, 3011-3025. Online: https: / / bit.ly/2RYbo4g.

Gerrits, M. (2010). «The role of interception in the hydrological cycle». Doctoral thesis. Delft, Netherlands: Technische Universiteit Delft. Online: https://bit.ly/3omnEr7.

Gomez, D. y col. (2008). «Rainfall and cloud-water interception in tropical montane forests in the eastern Andes of Central Peru». En: Forest Ecology and Management 255.3-4, 1315-1325. Online: https://bit.ly/3tWXhJx.

Gosling, W. y col. (2009). «Long-term drivers of change in Polylepis woodland distribution in the central Andes». En: Journal of Vegetation Science 20.6, 1041-1052. Online: https : / / bit . ly / 3eXxCfQ.

Harden, C. y col. (2013). «Effects of land-use change on water in Andean páramo grassland soils». En: Annals of the Association of American Geographers 103.2, 375-384. Online: https : / / bit . ly / 2RuPGVp.

Hernández, M., D. Granados y A. Sánchez (2003). «Productividad de los ecosistemas en las regiones áridas». En: Revista Chapingo. Serie Ciencias Forestales y del Ambiente 9.2, 113-123. Online: https:/ / bit.ly/33O6XLO.

Herzog, S. y col. (2002). «Ecology and conservation of High-Andean Polylepis forests». En: Ecotropica 8, 93-95. Online: https:/ / bit.ly/3hB4WL0.

Hoch, G. y C. Körner (2005). «Growth, demography and carbon relations of Polylepis trees at the world's highest treeline». En: Functional Ecology 19.6, 941-951. Online: https:/ / bit.ly/3fjdzHD.

Holwerda, F., F. Scatena y L. Bruijnzeel (2006). «Throughfall in a Puerto Rican lower montane rain forest: A comparison of sampling strategies». En: Journal of Hydrology 327.3-4, 592-602. Online: https:/ / bit.ly/3ymj11O.

Jadán, O. y col. (2019). «Regeneración de árboles en ecosistemas naturales y plantaciones de $\mathrm{Pi}$ nus patula (Pinaceae) dentro de un gradiente altitudinal andino (Azuay, Ecuador)». En: Revista de Biología Tropical 67.1, 182-195. Online: https: //bit.ly/3f12psa.

Jennings, S., N. Brown y D. Sheil (1999). «Assessing forest canopies and understorey illumination: canopy closure, canopy cover and other measures». En: Forestry: An International Journal of Forest Research 72.1, 59-74. Online: https: / / bit. ly/2RwoANR.

Johnson, M. y J. Lehmann (2006). «Doublefunneling of trees: Stemflow and root-induced preferential flow». En: Ecoscience 13.3, 324-333. Online: https://bit.ly/3tUJzqG.

Keim, R., A. Skaugset y M. Weiler (2005). «Temporal persistence of spatial patterns in throughfall». En: Journal of Hydrology 314.1-4, 263-274. Online: https:/ / bit.ly/3wd4CGD.

Kessler, M. (2002). «The "Polylepis problem": where do we stand». En: Ecotropica 8.2, 97-110. Online: https://bit.ly/3ymoHw0.

Lemmon, P. (1956). "A spherical densiometer for estimating forest overstory density». En: Forest science 2.4, 314-320. Online: https : / / bit. ly / 3eNKKDW.

- (1957). «Using Forest Densiometers». En: Journal of Forestry 55.9, 1-2. Online: https: / / bit.ly / 33LBfyP.

Levia, D. y E. Frost (2006). «Variability of throughfall volume and solute inputs in wooded ecosystems». En: Progress in Physical Geography 30.5, 605-632. Online: https://bit.ly/2RV0MmL.

Levia, D. y S. Herwitz (2005). «Interspecific variation of bark water storage capacity of three deciduous tree species in relation to stemflow yield and solute flux to forest soils». En: Catena 64.1, 117-137. Online: https://bit.ly/3eOMgFR.

Levia, D. y col. (2011). «Forest Hydrology and Biogeochemistry». En: Springer. Cap. Throughfall and Stemflow in Wooded Ecosystems, 425-443. Online: https://bit.ly/3ynopVt. 
Lieberman, M., D. Lieberman y R. Peralta (1989). «Forests are not just Swiss cheese: canopy stereogeometry of non-gaps in tropical forests». En: Ecology 70.3, 550-552. Online: https: / / bit.ly / 3w4TN9i.

Llorens, P. y F. Gallart (2000). «A simplified method for forest water storage capacity measurement». En: Journal of hydrology 240.1-2, 131-144. Online: https://bit.ly/3uREiBH.

Loescher, H., J. Powers y S. Oberbauer (2002). «Spatial variation of throughfall volume in an oldgrowth tropical wet forest, Costa Rica». En: Journal of Tropical Ecology, 397-407. Online: https: / / bit.ly/3bwU8d7.

Macinnis, C. y col. (2014). «Throughfall and stemflow vary seasonally in different land-use types in a lower montane tropical region of Panama». En: Hydrological Processes 28.4, 2174-2184. Online: https://bit.ly/2Rne4bB.

Moliĉová, H. y P. Hubert (1994). «Canopy influence on rainfall fields' microscale structure in tropical forests». En: Journal of Applied Meteorology 33.12, 1464-1467. Online: https:/ / bit.ly/3ydzWXB.

Molina, A. y col. (2019). «Contributions of throughfall, forest and soil characteristics to near-surface soil water-content variability at the plot scale in a mountainous Mediterranean area». En: Science of the Total Environment 647, 1421-1432. Online: https://bit.ly/3fqMMZX.

Montalvo, J. y col. (2018). «Características morfológico-funcionales, diversidad arbórea, tasa de crecimiento y de secuestro de carbono en especies y ecosistemas de Polylepis del sur de Ecuador». En: Ecología Austral 28.1-bis, 249-261. Online: https:/ / bit.ly/33KSQXI.

Morales, L. y col. (2018). «Differential seedling regeneration patterns across forest-grassland ecotones in two tropical treeline species (Polylepis spp.)» En: Austral Ecology 43.5, 514-526. Online: https://bit.ly/3ykOMLS.

Moser, G., D. Hertel y C. Leuschner (2007). «Altitudinal change in LAI and stand leaf biomass in tropical montane forests: a transect study in Ecuador and a pan-tropical meta-analysis». En: Ecosystems 10.6, 924-935. Online: https:/ / bit.ly / 33NC6z3.

Murakami, S. (2006). "A proposal for a new forest canopy interception mechanism: Splash droplet evaporation». En: Journal of Hydrology 319.1-4, 72-82. Online: https://bit.ly/3yfx6Bn.
Nadkarni, N. y M. Sumera (2004). «Old-growth forest canopy structure and its relationship to throughfall interception». En: Forest Science 50.3, 290-298. Online: https:/ / bit.ly/3tTlMr0.

Nisbet, T. (2005). «Water Use by Trees». En: Forestry Commision 65, 1-8. Online: https : / / bit . ly / 33Lk14T.

Norman, J. y G. Campbell (1989). «Plant physiological ecology». En: Springer. Cap. Canopy structur, 301-325. Online: https: / / bit.ly /2SLIwfO.

Ochoa, A., P. Crespo y R. Célleri (2018). «Quantification of rainfall interception in the high Andean tussock grasslands». En: Ecohydrology 11.3, e1946. Online: https:/ / bit.ly/33KbQpo.

Ochoa, A. y col. (2019). «Actual evapotranspiration in the high Andean grasslands: A comparison of measurement and estimation methods». En: Frontiers in Earth Science 7, 1-16. Online: https: //bit.ly/3ols2GS.

Oyarzún, C. y col. (2011). «Seasonal and annual throughfall and stemflow in Andean temperate rainforests». En: Hydrological Processes 25.4, 623-633. Online: https:/ / bit.ly/3hCi7vc.

Padrón, R. y col. (2015). «Rainfall in the Andean Páramo: new insights from high-resolution monitoring in Southern Ecuador». En: Journal of Hydrometeorology 16.3, 985-996. Online: https:/ / bit. ly/3yfFYHa.

Park, A. y J. Cameron (2008). «The influence of canopy traits on throughfall and stemflow in five tropical trees growing in a Panamanian plantation». En: Forest Ecology and Management 255.5-6, 1915-1925. Online: https://s.si.edu/2RYcVqS.

Pinos, J. (2014). «Biomasa foliar, desfronde y descomposición de la hojarasca en los rodales de Polylepis reticulata del Parque Nacional Cajas». Tesis de grado. Cuenca, Ecuador: Universidad de Cuenca. Online: https:/ / bit.ly/3uRJg1d.

Pinos, J. y col. (2017). «Leaf Litterfall and Decomposition of Polylepis reticulata in the Treeline of the Ecuadorian Andes». En: Mountain Research and Development 37.1, 87-96. Online: https: / / bit.ly / 3hARMxX.

Pukkala, T. y col. (1991). «Predicting spatial distribution of direct radiation below forest canopies». En: Agricultural and Forest Meteorology 55.3-4, 295-307. Online: https : / / bit . ly / 33LmEnh.

Quiroz, C. y col. (2019). "Comparison of Natural Regeneration in Natural Grassland and Pine Plantations across an Elevational Gradient in 
the Páramo Ecosystem of Southern Ecuador». En: Forests 10.9, 1-30. Online: https: / / bit.ly / 2SVwUHl.

Ramos Franco, A. y D. Armenteras (2019). «Interceptación y escorrentía del bosque altoandino en la reserva forestal protectora "El Malmo"». En: Acta Biológica Colombiana 24.1, 97-108. Online: https://bit.ly/3hruCKc.

Rangel, O. y H. Arellano (2010). «Colombia Diversidad Biótica X. Cambio Global (Natural) y Climático (Antrópico) en el páramo colombiano. Bogotá». En: Instituto de Ciencias NaturalesFacultad de Ciencias-Universidad Nacional de Colombia. Cap. Bosques De Polylepis: Un Tipo de vegetación condenado a la extinción, 443-478. Online: https:/ / bit.ly/3yoWpki.

Renison, D., I. Hensen y R. Suarez (2011). «Landscape structural complexity of high-mountain Polylepis australis forests: a new aspect of restoration goals». En: Restoration Ecology 19.3, 390-398. Online: https:/ / bit.ly/2RlsUze.

Renison, D. y col. (2006). "Cover and growth habit of Polylepis woodlands and shrublands in the mountains of central Argentina: human or environmental influence?» En: Journal of Biogeography 33.5, 876-887. Online: https: / / bit.ly / 3ojlLM6.

Roth, B., K. Slatton y M. Cohen (2007). «On the potential for high-resolution lidar to improve rainfall interception estimates in forest ecosystems». En: Frontiers in Ecology and the Environment 5.8, 421-428. Online: https://bit.ly/2QsQda8.

Staelens, J. y col. (2006). «Spatial variability and temporal stability of throughfall deposition under beech (Fagus sylvatica L.) in relationship to canopy structure». En: Environmental Pollution 142.2, 254-263. Online: https:/ / bit.ly/33LDl1R.

Teale, N. y col. (2014). «Impacts of vegetation and precipitation on throughfall heterogeneity in a tropical pre-montane transitional cloud forest». En: Biotropica 46.6, 667-676. Online: https: / / bit. ly/3hzDlKj.
Tinoco, B. y col. (2013). «Influence of patch factors and connectivity on the avifauna of fragmented Polylepis forest in the Ecuadorian Andes». En: Biotropica 45.5, 602-611. Online: https: / / bit.ly / 2RmA4mY.

Tsiko, C. y col. (2012). «Measuring forest floor and canopy interception in a savannah ecosystem». En: Physics and Chemistry of the Earth, Parts A/B/C 47, 122-127. Online: https:/ / bit.ly/2RmAgTe.

Valencia, B. y col. (2018). «Polylepis woodland dynamics during the last 20,000 years». En: Journal of Biogeography 45.5, 1019-1030. Online: https: / / bit.ly/33UhWn1.

Weiqing, Z. y col. (2007). «Spatial variability of throughfall in a Chinese pine (Pinus tabulaeformis) plantation in northern China». En: Frontiers of Forestry in China 2.2, 169-173. Online: https: / / bit.ly/3uUfo4b.

Wullaert, H. y col. (2009). «Spatial throughfall heterogeneity in a montane rain forest in Ecuador: extent, temporal stability and drivers». En: Journal of Hydrology 377.1-2, 71-79. Online: https: / / bit.ly/3eRSBk1.

Zhang, G. y col. (2006). «Modelling and measurement of two-layer-canopy interception losses in a subtropical evergreen forest of central-south China». En: Hydrology and Earth System Sciences 10.1, 65-77. Online: https:/ / bit.ly/3eOg71c.

Zimmermann, A., W. Wilcke y H. Elsenbeer (2007). «Spatial and temporal patterns of throughfall quantity and quality in a tropical montane forest in Ecuador». En: Journal of Hydrology 343.1-2, 80-96. Online: https://bit.ly/3tS49rM.

Zimmermann, A., B. Zimmermann y H. Elsenbeer (2009). «Rainfall redistribution in a tropical forest: Spatial and temporal patterns». En: Water Resources Research 45.11, 1-18. Online: https: / / bit.ly/3huuc5H.

Zimmermann, A. y col. (2008). «Spatio-temporal patterns of throughfall and solute deposition in an open tropical rain forest». En: Journal of Hydrology 360.1-4, 87-102. Online: https: / / bit.ly / 3hwkrnt. 